\title{
Expert Discussion: Predictive Markers
}

\author{
Angelo Paradiso ${ }^{a} \quad$ Giovanni Codacci-Pisanellib $^{b}$ Elisabetta Munzone ${ }^{c}$ \\ Hope S. Rugo ${ }^{d}$ Pierre Etienne Heudel ${ }^{e}$ \\ ${ }^{a}$ Experimental Oncology, Istituto Tumori G. Paolo II, IRCCS, Bari, Italy; ${ }^{b}$ Department of Medical and Surgical \\ Sciences and Biotechnology, University of Rome la Sapienza, Latina, Italy; ${ }^{\mathrm{C}}$ Division of Medical Senology, \\ European Institute of Oncology, IRCCS, Milan, Italy; ${ }^{\mathrm{d} B r e a s t}$ Oncology and Clinical Trials Education, University of \\ California San Francisco Comprehensive Cancer Center, San Francisco, CA, USA; ${ }^{\mathrm{e}}$ Medical Oncology Department, \\ Centre Léon-Bérard, Lyon, France
}

\section{Paradiso: PARP Inhibitors and BRCA Status in Early Breast Cancer. What Are the Current Criteria to Test for BRCA1/2-Mutation, What Would You Expect in the Future?}

Codacci-Pisanelli: I think the role of BRCA (breast cancer gene) mutations for PARP (poly-ADP-ribose polymerase) inhibitors in breast cancer is as confused as it is in ovarian cancer where some inhibitors are only approved for mutated cases while others are approved independently of the presence of a mutation. This seems to indicate that the presence of a BRCA mutation may play a role, but probably does not tell the whole story.

I expect that having lost its role in the prescription of PARP inhibitors BRCA testing will decrease to a more acceptable level, and it will be requested based only on the basis of a possible mutation based on personal/familial history.

Munzone: Testing for BRCA1/2 germline mutations has an established predictive role in breast cancer risk assessment, but recently, studies have also identified BRCA1/2 status as clinically relevant to selecting the most appropriate therapy for patients with early breast cancer.

Generally, testing for BRCA is offered to women with breast cancer who are younger than 50 years of age, have bilateral cancer, or have a significant family history.

Following promising results from the Olympia study presented at the ASCO meeting in June 2021, it was reported that for patients with early-stage, HER2-negative breast cancer with a high risk of recurrence and pathogenic or probably pathogenic BRCA1 or BRCA2 germline variants, 1 year of adjuvant olaparib should be offered after completion of (neo)adjuvant chemotherapy and local treatment.

The Olympia results highlight the need for genetic testing for BRCA mutations in patients diagnosed with early-stage high-risk breast cancer. These findings could have an important impact on treatment decisions for this patient population, possibly including the use of a PARP inhibitor in the adjuvant setting.

Rugo: At the present time, the guidelines still recommend using a cutoff age of 60 for testing. However, with the recent data demonstrating marked efficacy of olaparib in high-risk early-stage breast cancers associated with a BRCA mutation in the Olympia trial, there has been interest in expanding the guidelines for testing. In addition, both olaparib and talazoparib have demonstrated efficacy in BRCA-associated metastatic breast cancer. I expect that we will test judiciously in the future in patients not covered by the guidelines, including those with highrisk early-stage disease, and those with metastatic disease where next-generation sequencing (NGS) will easily pick up both germline and somatic mutations.

Heudel: In June 2021, Tutt et al. [1] published results of the Olympia trial where 1 year of adjuvant olaparib was associated with significantly longer survival free of local or distant disease in patients with high-risk early-stage HER2-negative breast cancer and germline BRCA mutations. Although very encouraging, there are many limits to these results. Indeed, this is only an interim analysis with a median follow-up of only 2.5 years; the study was randomized against placebo whereas adjuvant capecitabine is the standard treatment in the event of complete histological nonresponse after neoadjuvant 
chemotherapy, and finally the 3-year estimated overall survival was greater with olaparib, but the difference was not statistically significant. Considering these elements and the fact that in this study, it did not evaluate the effect of olaparib in hereditary forms of breast cancer other than those associated with the germline mutations BRCA1 or BRCA2, to date, there is no reason to expand the existing search criteria to all our patients with high-risk earlystage HER2-negative breast cancer. This clinical study further highlights the importance of researching hereditary forms of cancer in our patients by respecting the classic risk assessment criteria based on individual characteristics and family neoplastic history [2].

\section{Paradiso: What Is the Current Role of PD-L1 Testing in Breast Cancer, Which Tests/Scores Should Be Favored?}

Codacci-Pisanelli: Testing of PDL-1 (programmed cell death 1 ligand 1) presents several technical problems in most tumors, and this is a very relevant issue due to the need to select patients who have the best chance of responding to immune-stimulating antibodies and, on the opposite side, to identify those that are less likely to respond and may therefore be spared the toxic side effects (which can be serious).

Concerning breast cancer the IMpassion 130 trial used a scoring system that at the moment represents the standard in this setting: even if I think that when transferring data obtained in clinical trials to everyday practice we should maintain the original settings as much as possible, I also expect that in the future we could choose different tests that might prove more reproducible in nonspecialized centers.

Munzone: At present, patients with triple negative breast cancer (TNBC) with immunohistochemical expression of PD-L1 on tumor-associated immune cells derive a survival benefit from the addition of anti-PD-L1 therapy (atezolizumab) to nab-paclitaxel.

There are several PD-L1 assays to be used with each anti-PD-L1 drug. The immunohistochemical assay includes an antibody clone (22C3, 28-8, SP142, SP263, and 73-10) for use with a specific detection platform (DAKO Autostainer Link 48 platform, Agilent Inc. and Ventana BenchMark Ultra platform, Ventana Medical Systems Inc.).

The sensitivity of the PD-L1 detection kit varies depending on whether the assay is performed on tumor cells or immune cells.

The scoring algorithm and cutoffs for positivity were developed together with each corresponding anti-PD-L1/ PD-1 clinical program. The determination of the assay cutoffs correlated with the clinical activity of those agents, which is the basis for the accompanying diagnostics specified for each indication.

Expert Discussion: Predictive Markers
PD-L1 has demonstrated its utility as a biomarker that can now be used to identify patients who are more likely to respond to treatment with atezolizumab plus nab-paclitaxel or pembrolizumab plus chemotherapy for metastatic TNBC.

Rugo: PD-L1 testing is performed on tumor samples from patients with metastatic TNBC in order to assess potential efficacy of checkpoint inhibitors. Two tests have regulatory approval in the united states. The SP142 antibody evaluates PD-L1 in immune cells only, with $1 \%$ or greater defined as positive. This test is used to determine patients who could benefit from the combination of atezolizumab and nab-paclitaxel. The combined positive score is a score using the $22 \mathrm{C} 3$ antibody. A score of 10 or greater is defined as positive, and this test is used to determine patients who could benefit from the combination of pembrolizumab with either paclitaxel, nab-paclitaxel, or gemcitabine plus carboplatin. The choice of the PD-L1 assay should be directed by the proposed checkpoint inhibitor and paired chemotherapy. It is important to understand that there is incomplete overlap between these two assays.

Heudel: PD-1/PD-L1 has emerged as a promising therapeutic target in breast cancer subtypes, and Atezolizum$\mathrm{ab}$ and Pembrolizumab are both approved in metastatic TNBC $[3,4]$. Arezolizumab requires a PD-L1 positivity on immune cells of $1 \%$ (defined by the area occupied by PD-L1-positive immune cells over the entire tumor area). The PD-L1 test is a global issue with various tests, antibody types, scoring methods, and cutoffs. In the approval study for atezolizumab in metastatic TNBC, the immune cell score was assessed using the Ventana PD-L1 SP142 assay [5]. Depending on the country and the laboratory, there are other tests, but this still requires additional studies to ensure the quality of the results and a certain harmonization. Concerning pembrolizumab in the Keynote-173 study, PD-L1 status was defined according to a combined positive score (number of PD-L1-positive cells (tumor and immune cells) divided by the total number of tumor cells multiplied by 100 . However, in the Keynote-522 trial, the clinical benefit of pembrolizumab seems independent of this composite score, which proves the need to continue ancillary research in order to better select patients who may benefit from immunotherapy [6].

\section{Paradiso: How Should We Deal with PIK3CA Mutation Analyses. Whole Gene Analyses or Only Three Hot Spots in Exon 9 and Exon 20? Differences of Clinical Impact in the EU and USA?}

Codacci-Pisanelli: Specific inhibitors of PIK3CA are active in the presence of a mutated gene: data indicate that in most cases mutations can be mapped to specific 
hotspots that can be studied using PCR techniques. In the last few years, the widespread use of NGS has been supported by its technical development and by a sharp decrease in costs. NGS certainly provides data on the whole gene, but this requires validation in clinical settings in order to identify relevant mutations that result in drug sensitivity. It is certainly possible that, similar to what is happening in lung cancer, the availability of more genomic data will lead to the identification of PIK3CA inhibitors that may overcome resistance to first-generation inhibitors.

Munzone: In hormone receptor-positive breast cancer, the most common alteration in the Phosphatidylinositol 3-kinase (PI3K)-AKT-mTOR pathway is represented by PIK3CA mutations associated with resistance to endocrine therapy and disease progression.

These mutations can occur in several domains of the p110a catalytic subunit, but most are found in exons 9 and 20 which represent the "hot spots."

Recently, the PI3Ka-selective inhibitor alpelisib was the first PI3K inhibitor approved for clinical use in hormone receptor-positive metastatic breast cancer based on the results of the phase III SOLAR-1 trial. Several methods to assess PIK3CA mutational status in tumor samples have been developed and validated, including real-time polymerase chain reaction (PCR), digital droplet PCR, BEAMing assays, Sanger sequencing, and NGS panels.

Once alpelisib becomes widely available in the clinic, it is critical to adopt the best methodology to evaluate all possible gene alterations in order to increase the number of patients potentially benefiting from such treatments. In daily practice, accompanying diagnostic PCR may generally be sufficient to identify patients eligible for alpelisib treatment. However, the use of larger NGS panels, which are even more readily available at significantly reduced costs, could allow the identification of alterations in genes related to therapeutic resistance as well as less common PIK3CA mutations.

Diagnostic assays for assessment of PIK3CA mutation status are available and can be used on both tumor tissue and ctDNA. The latter is particularly valuable when tissue material is unavailable or inaccessible.

The US Food and Drug Administration (FDA) has approved alpelisib together with the therascreen PIK3CA RGQ PCR kit (QIAGEN GmbH), FoundationOne ${ }^{\circledR}$ CDx and FoundationOne ${ }^{\circledR}$ Liquid CDx as companion diagnostics. In contrast, the European Medicines (EMA) has generally recommended the use of a validated test for PIK3CA mutation evaluation.

Rugo: The SOLAR-1 trial evaluated efficacy of the a-specific PI3K inhibitor alpelisib in the major activating hot spots in exons 9 and 20. However, multiple other potential activating mutations may be detected, and a realworld analysis suggested that patients with these muta- tions could have similar progression-free survival compared to what was seen in SOLAR-1 - although this is purely comparative. In addition, other targetable mutations might be found using NGS rather than focused testing for just the three hot spots. Therefore I would favor whole gene analysis in order to most broadly define the potential benefit from targeted agents in the metastatic setting.

Heudel: PI3K is a targetable enzyme, in particular by its p110a isoform (catalytic subunit) which has activating mutations in $30 \%$ of hormone receptor-positive metastatic breast cancer. Despite the significant scientific rationale and numerous clinical trials, the development of PI3K inhibitors is undergoing complicated development due to an unfavorable therapeutic index, in particular for pan-PI3K inhibitors (for example buparlisib or pictilisib), responsible for excessive adverse events. The second generation of $\mathrm{PI} 3 \mathrm{~K}$ inhibitors is more selective for specific isoforms of PI3K and more specifically p110 $\alpha$ in metastatic breast cancer. The phase III SOLAR-1 trial (alpelisib) and SANDPIPER trial (taselisib) showed very interesting results in terms on efficacy in PI3K-mutant tumors $[7,8]$. Despite increased isoform specificity, adverse events remain clinically significant and dose reductions following protocol guidelines were associated with decreased efficacy [9]. This correlation between dose intensity, efficacy, and toxicity proves the importance of developing the most specific PI3K inhibitors possible and therefore, to date, that it is more important to analyze the main exons (9 and 20) of PIK3CA mutations than the whole gene.

\section{Paradiso: Is There a Role for ESR1 Mutation Analysis in Breast Cancer?}

Codacci-Pisanelli: Hormonal treatment is very effective in breast cancer, and even more so since new agents (CDK-2 and PIK3Ca inhibitors) have been added to associated aromatase inhibitors and to fulvestrant. Unfortunately, resistance remains the main problem, and a more detailed analysis of estrogen receptors could provide elements to overcome it.

At the moment several reports suggest that mutations in ESR1 may be responsible for clinical resistance to hormonal treatment and, more importantly, to the new combinations, but I think further experiments are needed before we can apply these analyses in the clinic.

This however shows how the application of sequencing techniques to large genome segments, that is made possible by NGS, may provide relevant information in many aspects of cancer treatment.

Munzone: ESR1 mutations have recently emerged as a key mechanism of aromatase inhibitor (AI) resistance in 
estrogen receptor-positive metastatic breast cancer and for which specific drugs are under development. ESR1 mutations are a novel prognostic factor of poor survival and can be detected by analyzing blood samples.

Clonal selection for ESR1 hot spot mutations can occur in the early stages of the disease. These mutations may emerge after or during adjuvant or neoadjuvant endocrine therapy.

ESR1 mutations are rare in primary breast cancer, with a rate of less than $5 \%$. However, in advanced breast cancer, ESR1 mutations occur at a frequency of $20-40 \%$, depending on the analysis techniques, and in relation to the number of treatment lines received.

New selective estrogen receptor modulators or degraders (with or without CDK4/6 inhibition) or highdose tamoxifen or fulvestrant may represent possible therapeutic strategies to overcome resistance related to ESR1 mutations.

Preclinical studies of these drugs have demonstrated efficacy in inhibiting tumor cell growth in models with ESR1 mutations. These novel treatments may overcome resistance related to ESR1 mutations, potentially offering a new therapeutic option for patients with estrogen receptor-positive/HER2-negative metastatic breast cancer in the future.

Rugo: ESR1 mutations clearly predict resistance to aromatase inhibitors, and some mutations predict less responsiveness to the selective estrogen receptor downregulatory fulvestrant. ESR1 mutations are uncommon at the initial presentation and increase under treatment with aromatase inhibitors and even during treatment with fulvestrant. This mutation can also become undetectable during treatment. Although this mutation has predictive power, this analysis does not impact therapy in most settings. in the first-line setting without immediate prior AI, an AI plus CDK4/6 inhibitor is a reasonable choice without testing. In the second-line setting, fulvestrant is the common choice, so ESR1 testing would not inform this choice. In the third- or greater-line setting, ESR1 analysis might be helpful if exemestane plus everolimus was a potential option.

Heudel: In hormone receptor-positive metastatic breast cancer, ESR1 mutations are a common cause of acquired resistance to AIs. ESR1 mutations are detected in between 1 and $5 \%$ of patients upon first relapse but in up to $40 \%$ of metastatic breast cancer patients who become resistant to an AI. The simplest mechanism by which ESR1 mutations produce resistance is the lifelong constitutive activity that keeps estrogen receptors unaffected by AI depletion of estrogen. In view of this biological mechanism, several retrospective studies have evaluated the interest of selective estrogen receptor modulators (such as tamoxifen) and selective estrogen receptor degraders (such as fulvestrant) with en- couraging results [10]. To date, prospective studies are rare but the phase III PADA-1 trial has nevertheless shown that the presence of an ESR1 mutation at baseline leads to a worse prognosis but that the disappearance of the mutation (on cell-free DNA) after 1 month of letrozole + palbociclib improved the prognosis of patients initially positive for ESR1 [11]. This clinical trial will also allow us to respond to the benefit of replacing an anti-aromatase with fulvestrant in the event of the appearance of an ESR1 mutation and thus to better specify the role and the time of ESR 1 mutation analysis in our clinical practice.

\section{Paradiso: Which Other Molecular Predictive Tests Including NGS on Primary Tumor or Liquid Biopsy Would You Consider in Metastatic Breast Cancer Patients?}

Codacci-Pisanelli: Liquid biopsy is certainly very appealing due to its limited invasiveness, but its practical application outside clinical trials performed in large cancer centers presumably implies that it may be very demanding from a technical point of view.

NGS promises to provide an enormous amount of data, but until now my impression is that this does not translate into equivalent identification of targets, so I think more specific techniques (such as droplet PCR) maintain their role in evaluating mutations in previously identified genes.

Let me add that I think it would be important to focus on "very aggressive" breast cancer: patients with disease that progresses despite every treatment. These cancers, these patients, require a special effort in order to identify specific targets and hopefully an effective treatment.

Munzone: The two FDA-approved molecular profiling NGS assays that interrogate more genes at once are the Foundation One CDx assay and the MSK-IMPACT assay. Other NGS tests are under development. In 2019, the FDA also granted Breakthrough Device Designation for Illumina's pan-cancer test, TruSight Oncology 500, which uses DNA and RNA from tumor samples to identify small DNA variants, fusions and splice variants, as well as tumor mutational burden and microsatellite instability.

Certainly, there is an urgent need to coordinate and homologize analyses and reports of NGS results in order to perform real-world observational studies that collect the experience of a very large number of patients, and to establish the real utility of these gene profiles according to ESMO and FDA criteria.

Interdisciplinary discussion in multidisciplinary teams such as Molecular Tumor Boards is very important to help interpret the unclear molecular results often seen with NGS testing. 
Important unresolved issues that will need to be addressed in the future include deciding what is the best tissue to perform NGS (primary tumor vs. metastases, tumor DNA vs. circulating tumor DNA), when is the right time to test (at the first diagnosis of advanced disease or when the disease is refractory), and whether there are clinical trial designs that use NGS and allow for the use of control groups. Finally, the use of adequate informed consent before NGS testing and the communication of NGS test results to patients are two very important issues that have raised ethical debates.

Advances in genomic techniques have led to improvements in the isolation and detection of circulating tumor DNA (ctDNA), a component of a peripheral blood collection/liquid biopsy. Liquid biopsy offers a minimally invasive method to collect representative genetic information of the primary tumor and/or site of metastasis and thus may provide valuable information for potential targeted therapies and tumor surveillance methods. However, the lack of prospective clinical trials demonstrating direct benefit to patients has limited, for the time being, the implementation of liquid biopsies in standard clinical applications.

Rugo: Other molecular predictive tests include tumor mutational burden, microsatellite instability, somatic BRCA1 or 2 mutations, somatic HER 2 mutations, and the NKTR fusion gene. All of these findings have been associated with specific therapies that have demonstrated efficacy. Other findings may qualify patients for clinical trials, for example, FGFR mutations.

Heudel: In daily clinical practice, NGS is frequently used, either by whole genome sequencing, whole exome sequencing, or multigene assays. From a tumor biopsy or even a liquid biopsy (circulating cell-free DNA/RNA or circulation tumor cells), the gene panels must allow the detection of actionable molecular targets guiding the therapeutic strategy. To date, international guidelines do not include multigene testing in the management of patients with metastatic breast cancer. Concerning metastatic TNBC patients with limited standard-of-care options, using multigene testing can identify new molecularly targeted treatment indications or helps in the selection of patients who can be included in clinical trials [12-15]. It is therefore preferable to carry out these molecular analyses as soon as possible in the management of these patients with limited therapeutic resources.

\section{Conflict of Interest Statement}

Angelo Paradiso: no conflict of interest to disclose. Giovanni Codacci-Pisanelli: no conflict of interest to disclose. Elisabetta Munzone: consulting or advisory role for Genomic Health, Pierre Fabre, and Eisai. Hope S. Rugo: research support for clinical trials through the University of California to Pfizer, Merck, Novartis,
Lilly, Roche, Odonate, Daiichi, Seattle Genetics, Macrogenics, Sermonix, Astra Zeneca, OBI and Gilead, Ayala; honoraria: Puma, Mylan, Samsung. Pierre Etienne Heudel: grants, personal fees, and nonfinancial support from Pfizer, grants and nonfinancial support from Novartis, grants and nonfinancial support from Roche, grants, personal fees, and nonfinancial support from Astra Zeneca, personal fees and nonfinancial support from Mylan, grants, personal fees, and nonfinancial support from Pierre Fabre, personal fees and nonfinancial support from Amgen and personal fees and nonfinancial support from Seagen.

\section{Chair}

Prof. Angelo Paradiso

Experimental Oncology

Istituto Tumori G. Paolo II, IRCCS

Via O. Flacco, 65

IT-70125 Bari (Italy)

a.paradiso@oncologico.bari.it

\section{Participants}

Prof. Giovanni Codacci-Pisanelli

University of Rome la Sapienza

Department of Medical and Surgical Sciences and

Biotechnology

Corso della Repubblica, 79

IT-04100 Latina (Italy)

giovanni.codacci-pisanelli@uniroma1.it

\section{Dr. Elisabetta Munzone}

Division of Medical Senology

European Institute of Oncology, IRCCS

Via Ripamonti, 435

IT-20141 Milano (Italy)

elisabetta.munzone@ieo.it

\section{Prof. Hope S. Rugo}

Breast Oncology and Clinical Trials Education

University of California San Francisco Comprehensive

Cancer Center

1825 4th Street

San Francisco, CA 94158 (USA)

Hope.Rugo@ucsf.edu

\section{Dr. Pierre Etienne Heudel, Msc, LLM}

Medical Oncology Department

Centre Léon-Bérard

28 , rue Laënnec

FR-69373 Lyon cedex 08 (France)

PierreEtienne.HEUDEL@lyon.unicancer.fr 


\section{References}

1 Tutt AN, Garber JE, Kaufman B, Viale G, Fumagalli D, Rastogi P, et al; OlympiA Clinical Trial Steering Committee and Investigators. Adjuvant olaparib for patients with BRCA1or BRCA2-mutated breast cancer. N Engl J Med. 2021 Jun;384(25):2394-405.

2 Paluch-Shimon S, Cardoso F, Sessa C, Balmana J, Cardoso MJ, Gilbert F, et al; ESMO Guidelines Committee. Prevention and screening in BRCA mutation carriers and other breast/ovarian hereditary cancer syndromes: ESMO clinical practice guidelines for cancer prevention and screening. Ann Oncol. 2016 Sep;27 suppl 5:v103-10.

3 Schmid P, Adams S, Rugo HS, Schneeweiss A, Barrios CH, Iwata $\mathrm{H}$, et al; IMpassion130 Trial Investigators. Atezolizumab and nab-paclitaxel in advanced triple-negative breast cancer. N Engl J Med. 2018 Nov;379(22):210821.

4 Schmid P, Salgado R, Park YH, MuñozCouselo E, Kim SB, Sohn J, et al. Pembrolizumab plus chemotherapy as neoadjuvant treatment of high-risk, early-stage triple-negative breast cancer: results from the phase $1 \mathrm{~b}$ open-label, multicohort KEYNOTE-173 study. Ann Oncol. 2020 May;31(5):569-81.

5 FDA approves atezolizumab for PD-L1 positive unresectable locally advanced or metastatic triple-negative breast cancer. FDA US Food and Drug [cited 2020 May 16]. Available from: https://www.fda.gov/drugs/drug-approvals-and-databases/fda-approves-atezoli- zumab-pd-11-positive-unresectable-locallyadvanced-or-metastatic-triple-negative.

6 Schmid P, et al. KEYNOTE-522: phase III study of neoadjuvant pembrolizumab + chemotherapy vs. placebo + chemotherapy, followed by adjuvant pembrolizumab vs. placebo for early-stage TNBC. Presented at: ESMO Virtual Plenary, July 15-16, 2021. Available from: https://www.esmo.org/meetings/pastmeetings/keynote-522-phase-iii-study-ofneoadjuvant-chemotherapy-vs-placebo-chemotherapy-followed-by-adjuvant-vs-placebo-for-early-stage-tnbc.

7 André F, Ciruelos EM, Juric D, Loibl S, Campone M, Mayer IA, et al. Alpelisib plus fulvestrant for PIK3CA-mutated, hormone receptor-positive, human epidermal growth factor receptor-2-negative advanced breast cancer: final overall survival results from SOLAR-1. Ann Oncol. 2021 Feb;32(2):208-17.

8 Dent S, Cortés J, Im YH, Diéras V, Harbeck N, Krop IE, et al. Phase III randomized study of taselisib or placebo with fulvestrant in estrogen receptor-positive, PIK3CA-mutant, HER2-negative, advanced breast cancer: the SANDPIPER trial. Ann Oncol. 2021 Feb; 32(2):197-207.

9 Rugo HS, André F, Yamashita T, Cerda H, Toledano I, Stemmer SM, et al. Time course and management of key adverse events during the randomized phase III SOLAR-1 study of PI3K inhibitor alpelisib plus fulvestrant in patients with HR-positive advanced breast cancer. Ann Oncol. 2020 Aug;31(8): 1001-10.

10 Brett JO, Spring LM, Bardia A, Wander SA. ESR1 mutation as an emerging clinical biomarker in metastatic hormone receptor-positive breast cancer. Breast Cancer Res. 2021 Aug;23(1):85.

11 Bidard FC, Callens C, Dalenc F, et al. Prognostic impact of ESR1 mutations in ER+ HER2- MBC patients with prior treatment with first-line AI and palbociclib: an exploratory analysis of the PADA-1 trial. ASCO20 Virtual Scientific Program. Abstract 1010.

12 Cobain EF, Wu YM, Vats P, Chugh R, Worden F, Smith DC, et al. Assessment of clinical benefit of integrative genomic profiling in advanced solid tumors. JAMA Oncol. 2021 Apr;7(4):525-33.

13 McKenzie AJ, H Dilks H, Jones SF, Burris H 3rd. Should next-generation sequencing tests be performed on all cancer patients? Expert Rev Mol Diagn. 2019 Feb;19(2):89-93.

14 Colomer R, Mondejar R, Romero-Laorden N, Alfranca A, Sanchez-Madrid F, QuintelaFandino M. When should we order a next generation sequencing test in a patient with cancer? E Clin Med. 2020 Jul;25:100487.

15 Stover DG, Reinbolt RE, Adams EJ, Asad S, Tolliver K, Abdel-Rasoul M, et al. Prospective decision analysis study of clinical genomic testing in metastatic breast cancer: impact on outcomes and patient perceptions. JCO Precis Oncol. 2019 Nov;3(3):1-11. 